\title{
Effects of Long-Term Combination Treatment with Valproate and Atypical Antipsychotics on Bone Mineral Density and Bone Metabolism in Premenopausal Patients with Bipolar Disorder: A Preliminary Study
}

\author{
Jaewon Yang, Sook-Haeng Joe ${ }^{凶}$, Moon-Soo Lee, Young-Hoon Ko, In-Kwa Jung and Seung-Hyun Kim \\ Department of Psychiatry, Korea University College of Medicine, Korea University Research Institute of Mental Health, Seoul, Korea
}

\begin{abstract}
Objective We investigated bone mineral density (BMD) and bone metabolism in female bipolar patients who were undergoing long-term treatment with valproate combined with a low-dose atypical antipsychotic.

Methods Nineteen premenopausal women with bipolar disorder who were treated with valproate combined with atypical antipsychotics for at least 2 years were evaluated. The BMD was measured at lumbar spine and femur sites using dual-energy X-ray absorptiometry (DEXA). The biochemical markers of bone turnover and circulating levels of gonadal hormones were assessed. Subjects with abnormal DEXA scans were compared to those with normal scans.

Results Nine (47\%) of nineteen subjects showed osteopenia or osteoporosis. The T-score for subjects with abnormal DEXA scans was -1.988. Decreased BMD was more prominent in the proximal femur than in the lumbar spine. Subjects with abnormal DEXA scans had high phosphorus and low testosterone levels relative to subjects with normal scans ( $\mathrm{p}=0.008$ and $\mathrm{p}=0.028$, respectively). There was a significant negative correlation between phosphorus, osteocalcin, and femur neck BMD ( $\mathrm{p}<0.05)$. However, multivariate analysis did not show a significant association between femur and lumbar BMD and biochemical markers of bone turnover.

Conclusion Long-term treatment with valproate combined with low-dose atypical antipsychotics may adversely affect BMD in premenopausal women with bipolar disorder. A prospective, controlled-study with a larger population is warranted, and assessment of BMD and bone metabolism should be taken into consideration in long-term therapy with valproate and atypical antipsychotics.
\end{abstract}

Psychiatry Investig 2011;8:256-261

Key Words Bipolar disorder, Bone density, Bone metabolism, Females, Valproate.

\section{INTRODUCTION}

Certain psychiatric disorders are known to be associated with low bone mineral density (BMD), including schizophrenia, schizoaffective states, and major depressive disorder. ${ }^{1-5}$ The nature of the relationship between psychiatric disorders and psychotropic medications and bone metabolism is unresolved; however, there is growing evidence that medications used to treat disorders are associated with low BMD or alter-

Received: September 4, 2010 Revised: November 24, 2010

Accepted: December 1, 2010 Available online: August 5, 2011

$\triangle$ Correspondence: Sook-Haeng Joe, MD, PhD

Department of Psychiatry, Korea University College of Medicine, Korea University Research Institute of Mental Health, 97 Gurodong-gil, Guro-gu, Seoul 152-703, Korea

Tel: +82-2-2626-3160, Fax: +82-2-852-1937, E-mail: shange@korea.ac.kr

(c) This is an Open Access article distributed under the terms of the Creative Commons Attribution Non-Commercial License (http://creativecommons.org/licenses/bync/3.0) which permits unrestricted non-commercial use, distribution, and reproduction in any medium, provided the original work is properly cited. ations in the neuroendocrine system that influences bone turnover. ${ }^{5}$ Several reports suggested that patients receiving longterm antipsychotics show reduced BMD. ${ }^{5,6}$ Low dietary calcium intake, smoking, polydipsia, and antipsychotic-related hyperprolactinemia have been implicated. ${ }^{3}$

In patients with bipolar disorder, several lines of evidence have suggested that lithium has a negative impact on bone metabolism with an association of hyperparathyroidism. ${ }^{5}$ However, there have been no reports examining the relationship between valproate as a mood stabilizer and BMD in bipolar disorder. ${ }^{5,7}$ A few studies have found that BMD was decreased in epileptic patients who received valproate..$^{8-11}$ Valproate can be used to treat both bipolar disorder and epilepsy; however, to our knowledge no study of the relationship between valproate and BMD in bipolar disorder has been reported. Valproate is generally the first drug of choice when selecting a mood stabilizer, however mood stabilizers are frequently combined 
with antipsychotic medications in clinical practice.

In this report, we investigated BMD and markers of bone metabolism in female bipolar patients treated with a long-term combination of valproate and low-dose atypical antipsychotics.

\section{METHODS}

\section{Subjects}

Nineteen subjects were eligible for participation. Subjects included in the study were 1) bipolar patients diagnosed according to the Diagnostic and Statistical Manual of Mental Disorders, Fourth Edition criteria; 2) patients receiving maintaining therapy with valproate and low-dose atypical antipsychotics for at least 2 years; 3 ) premenopausal women; and 4 ) ambulatory outpatients with normal activity and nutritionally adequate diets. Exclusions included 5) pregnant or breast-feeding women; 6) alcoholism or drug abuse; 7) comorbid medical diseases known to affect bone metabolism such as hepatic or renal disorders, hypothyroidism, or malabsorption; 8) a history of medication treatments that can affect bone turnover (e.g., vitamin $\mathrm{A}$, glucocorticoids, oral contraceptives, thiazides, calcitonin, vitamin D, calcium supplements, or biphosphonates). Written informed consent was obtained from all subjects after they were given information about the study. The Korea University Institutional Review Board approved this study.

\section{Clinical and biochemical evaluation}

Demographic and clinical data were collected including age of disease onset, duration of illness, and dose and duration of medication. The Brief Psychiatric Rating Scale, Young Mania Rating Scale, Hamilton rating scale for depression, and Clinical Global Impression scores were applied. Other variables included smoking, alcohol, coffee drinking, exercise, diet, history of fracture/fall, and menstrual irregularity. Bone formation and resorption markers were assessed for calcium, phosphorus, alkaline phosphatase, osteocalcin, 25( $\mathrm{OH})$-vitamin $\mathrm{D}$, parathyroid hormone (PTH), and urine deoxypyridinoline. Blood valproate levels, prolactin, and circulating levels of gonadal hormones including prolactin, estradiol, lutenizing hormone, follicle-stimulating hormone, progesterone and testosterone were also assessed.

\section{Bone mineral density measurement}

The BMD was measured at the lumbar spine and various femur sites using dual-energy X-ray absorptiometry (DEXA) (Hologic Discovery-A bone densitometer; Hologic, Inc., Bedford, MA, USA). T-scores and Z-scores suggested by World Health Organization criteria ${ }^{12}$ were calculated in all subjects. Osteopenia was defined as less than -1.0 and greater than -2.5 . Osteoporosis was defined as -2.5 or lower, meaning a bone density that was two and a half standard deviations below the mean of an average thirty-year-old woman. The Z-score was the number of standard deviations above or below the mean for ageand sex-matched controls.

\section{Statistical analysis}

Comparison of all demographic, biochemical parameters and densitometrical parameters were conducted using the Mann-Whitney $\mathrm{U}$ test between subjects with abnormal and normal DEXA scans. Nonparametric repeated measure analyses with Wilcoxon's signed rank post-hoc tests were applied for comparison between the BMD of femurs and lumbar spine. Correlation between clinical, biochemical and BMD measurements was tested by a Spearman rank correlation. Within groups, simple linear regression was used to determine the association between $\mathrm{BMD}$ and various risk factors including age, weight, duration of medication, alcohol, smoking, coffee, and daily exercise as well as biochemical indices of bone metabolism. Multiple linear regressions with significant variables were used to determine the relating factors. The SPSS 15.0 package program was used in the statistical analysis. A value of $\mathrm{p}<$ 0.05 was considered statistically significant.

\section{RESULTS}

Nine (47.4\%) out of all 19 subjects showed decreased BMD (osteopenia/osteoporosis defined as T-score <-1.0) on DEXA scans. Two of the nine subjects (22.2\%) had osteoporosis. Total body BMD of whole study subjects was $-0.95 \pm 1.19 \mathrm{~g} / \mathrm{cm}^{2}$, which was lower than the BMD mean value of adult Korean women shown in previous reports $\left(1.16 \pm 0.11 \mathrm{~g} / \mathrm{cm}^{2}\right.$ aged in their thirties and $1.08 \pm 0.14 \mathrm{~g} / \mathrm{cm}^{2}$ aged in their forties). The $\mathrm{T}$-scores of the right and left femurs were $-0.18 \pm 1.26$ and $-0.24 \pm 1.33$, respectively, and the T-score of the lumbar spine was $-0.30 \pm 1.22{ }^{13,14} \mathrm{~T}$-scores for subjects with abnormal DEXA scans were -1.988 compared to subjects with normal DEXA scans of -0.050 .

The mean age of subjects was $39.68 \pm 10.17$ years. The average body mass index (BMI) was in the overweight range at $24.33 \pm 3.63 \mathrm{~kg} / \mathrm{m}^{2}$. The antipsychotics used for concomitant treatment were risperidone at a dose range of $0.5-1.5 \mathrm{mg}(\mathrm{n}=$ 9), olanzapine with a dose range of $2.5-5 \mathrm{mg}(\mathrm{n}=4)$, and quetiapine with a dose range of $25-100 \mathrm{mg}(\mathrm{n}=6)$ (Table 1$)$.

BMD levels were found to be lower in the proximal femur (femur neck and trochanter) than in the lumbar spine ( $\mathrm{p}=$ 0.000). In comparison between both groups, femoral neck Zscores of subjects with abnormal DEXA scans were statistically lower than those of subjects with normal DEXA scans $(\mathrm{p}=0.021$ and $\mathrm{p}=0.001$, respectively). Z-scores for the trochanter (right and left) and lumbar spine were lower in subjects 
Table 1. Clinical characteristics of the study population

\begin{tabular}{|c|c|}
\hline Parameters & $\begin{array}{c}\text { Total subjects } \\
\qquad(\mathrm{N}=19)\end{array}$ \\
\hline Age (mean $\pm S D$, years) & $39.68 \pm 10.17$ \\
\hline Education (mean $\pm S D$, years) & $12.16 \pm 3.13$ \\
\hline Height $($ mean $\pm S D, c m)$ & $159.95 \pm 3.98$ \\
\hline Weight (mean $\pm \mathrm{SD}, \mathrm{kg}$ ) & $61.16 \pm 7.17$ \\
\hline $\mathrm{BMI}\left(\mathrm{mean} \pm \mathrm{SD}, \mathrm{kg} / \mathrm{m}^{2}\right)$ & $24.33 \pm 3.63$ \\
\hline \multicolumn{2}{|l|}{ Exercise* } \\
\hline Regular (N) & 7 \\
\hline $\operatorname{Irregular}(\mathrm{N})$ & 4 \\
\hline None $(\mathrm{N})$ & 8 \\
\hline Onset age (mean $\pm \mathrm{SD}$, years) & $31.84 \pm 9.92$ \\
\hline No. of episodes (mean \pm SD, N) & $3.79 \pm 2.02$ \\
\hline No. of admissions (mean \pm SD, $\mathrm{N}$ ) & $2.74 \pm 2.02$ \\
\hline Medication duration (mean $\pm \mathrm{SD}$, months) & $49.47 \pm 23.52$ \\
\hline $\mathrm{BPRS}($ mean $\pm \mathrm{SD})$ & $25.53 \pm 2.95$ \\
\hline YMRS (mean $\pm S D)$ & $3.37 \pm 2.45$ \\
\hline $\mathrm{HAMD}($ mean $\pm \mathrm{SD})$ & $8.41 \pm 6.35$ \\
\hline Valproate dose (mean $\pm \mathrm{SD}, \mathrm{mg} /$ day) & $527.78 \pm 157.39$ \\
\hline \multicolumn{2}{|l|}{ Concomitant antipsychotics } \\
\hline Risperidone, N (range) & $9(0.5-1.5 \mathrm{mg})$ \\
\hline Olanzapine, $\mathrm{N}$ (range) & $4(2.5-5 \mathrm{mg})$ \\
\hline Quetiapine, N (range) & $6(25-100 \mathrm{mg})$ \\
\hline
\end{tabular}

*regular exercise, 2 times or more/week, Irregular exercise, 1 time or less/week. BMI: Body Mass Index, BPRS: Brief Psychiatric Rating Scale, YMRS: Young Mania Rating Scale, HAMD: Hamilton Depression Rating Scale

with abnormal DEXA scans, however these differences did not have a statistical significance $(\mathrm{p}=0.360, \mathrm{p}=0.055$, and $\mathrm{p}=$ 0.101 , respectively). $\mathrm{BMD}$ values for the two groups are shown in Table 2.

When comparing the clinical and laboratory findings, no variables were different between the two groups for any of the following demographic and clinical parameters: marital status ( $\mathrm{p}=0.807)$, occupation ( $\mathrm{p}=0.489)$, family history $(\mathrm{p}=1.000)$, suicide history $(\mathrm{p}=1.000)$, exercise $(\mathrm{p}=0.581)$, alcohol $(\mathrm{p}=$ $1.000)$ or smoking $(\mathrm{p}=1.000)$. Subjects with abnormal DEXA scans tended to have longer medication lengths $(53.67 \pm 17.18$ vs. $42.70 \pm 28.48, \mathrm{p}=0.447$ ) although this was not significant. The mean values for all bone turnover biochemical markers were within normal ranges; however some values showed significant differences between the two groups (Table 3). Subjects showing abnormal DEXA scans had high phosphorus (3.99 vs. $3.32, \mathrm{p}=0.008)$, and low testosterone ( 0.20 vs. $0.49, \mathrm{p}=$ $0.028)$ levels relative to subjects with normal DEXA scans. Subjects with abnormal DEXA scans also showed increased levels of osteocalcin and deoxypyridinoline, but these did not
Table 2. BMD of the subjects with abnormal and normal DEXA scans

\begin{tabular}{lcc}
\hline Site of bone mass & $\begin{array}{c}\text { Abnormal DEXA } \\
\text { scans }(\mathrm{N}=9) \\
(\text { mean } \pm \text { SD })\end{array}$ & $\begin{array}{c}\text { Normal DEXA } \\
\text { scans }(\mathrm{N}=10) \\
(\mathrm{mean} \pm \mathrm{SD})\end{array}$ \\
\hline $\begin{array}{l}\text { Total body BMD } \\
\text { Femur, right }\end{array}$ & $-0.98 \pm 1.19$ & $-0.05 \pm 0.68$ \\
Neck & & \\
BMD $\left(\mathrm{g} / \mathrm{cm}^{2}\right)^{* * *}$ & $0.70 \pm 0.08$ & $0.87 \pm 0.098$ \\
Z-score** & $-0.06 \pm 0.94$ & $1.070 \pm 0.80$ \\
Trochanter & & \\
BMD $\left(\mathrm{g} / \mathrm{cm}^{2}\right)^{* * *}$ & $0.61 \pm 0.07$ & $0.72 \pm 0.05$ \\
Z-score & $0.60 \pm 1.64$ & $1.02 \pm 0.61$ \\
Total & & \\
BMD $\left(\mathrm{g} / \mathrm{cm}^{2}\right)^{* * *}$ & $0.81 \pm 0.10$ & $0.97 \pm 0.06$ \\
Z-score** & $0.10 \pm 1.01$ & $1.22 \pm 0.51$ \\
Femur, left & & \\
Neck & & $1.00 \pm 0.07$ \\
BMD $\left(\mathrm{g} / \mathrm{cm}^{2}\right)^{* * *}$ & $0.68 \pm 0.72$ & $0.85 \pm 0.86$ \\
Z-score** & $-0.25 \pm 0.74$ & $0.88 \pm 0.74$ \\
Trochanter & & $0.74 \pm 0.05$ \\
BMD $\left(\mathrm{g} / \mathrm{cm}^{2}\right)^{* *}$ & $0.62 \pm 0.07$ & $1.16 \pm 0.67$ \\
Z-score & $0.25 \pm 0.91$ & \\
Total & & \\
BMD $\left(\mathrm{g} / \mathrm{cm}^{2}\right)^{* * *}$ & $0.82 \pm 0.09$ & \\
Z-score** & $0.18 \pm 0.85$ & \\
Lumbar spine & & \\
BMD $\left(\mathrm{g} / \mathrm{cm}^{2}\right)^{* *}$ & $0.93 \pm 0.69$ & \\
Z-score & $0.13 \pm 0.92$ & \\
\hline
\end{tabular}

${ }^{* *} \mathrm{p}<0.01,{ }^{* * *} \mathrm{p}<0.001$. DEXA: Dual-energy X-ray absorptiometry, BMD: bone mineral density; defined according to the World Health Organization operational definition (i.e.) BMD T-score -2.5 for osteoporosis and a BMD T-score between 1 and -2.5 for osteopenia. Mann-Whitney U test

quite reach statistical significance. Serum $25(\mathrm{OH})$ vitamin D and PTH levels tended to be lower in abnormal DEXA scan subjects with no statistical significance.

There was a significant negative correlation between phosphorus level and both the left and right femur neck BMDs $(\mathrm{r}=-0.503,-0.552, \mathrm{p}<0.05)$. PTH had positive correlation with both femur neck BMDs ( $\mathrm{r}=0.501, \mathrm{r}=0.520, \mathrm{p}<0.05)$ and osteocalcin showed a negative correlation with the left femur neck $\operatorname{BMD}(r=0.548, p<0.05)$. However, disease-related factors (e.g., duration and severity of illness, number of episodes, and duration of valproate use) as well as demographic factors did not correlate with BMD of the femur and lumbar spine. Factors contributing to the variance in BMD were identified by subsets of linear regression. None of the identified factors, 
Table 3. Laboratory findings of the subjects with abnormal and normal DEXA scans

\begin{tabular}{|c|c|c|c|}
\hline Laboratory data & Reference range & $\begin{array}{c}\text { Abnormal DEXA scans }(\mathrm{N}=9) \\
(\operatorname{mean} \pm \mathrm{SD})\end{array}$ & $\begin{array}{c}\text { Normal DEXA scans }(\mathrm{N}=10) \\
(\operatorname{mean} \pm \mathrm{SD})\end{array}$ \\
\hline Calcium (ng/dL) & $8.5-10.5$ & $9.3 \pm 0.1$ & $9.4 \pm 0.3$ \\
\hline Phosphorus (ng/dL)* & $2.5-4.5$ & $4.0 \pm 0.5$ & $3.3 \pm 0.4$ \\
\hline ALP (IU/L) & $44-147$ & $60.8 \pm 17.2$ & $61.3 \pm 12.4$ \\
\hline $25(\mathrm{OH})$ vitamin $\mathrm{D}(\mathrm{ng} / \mathrm{mL})$ & $25-66$ & $22.8 \pm 5.9$ & $31.9 \pm 10.0$ \\
\hline Parathormone (pg/mL) & $6-40$ & $23.2 \pm 13.0$ & $29.2 \pm 11.4$ \\
\hline Osteocalcin (ng/mL) & $2.5-13$ & $6.0 \pm 3.0$ & $4.1 \pm 1.2$ \\
\hline Deoxypyridoline (ng/mL) & $3-8$ & $5.3 \pm 2.1$ & $4.4 \pm 1.0$ \\
\hline Prolactin (ng/mL) & $2.8-29$ & $28.4 \pm 27.9$ & $11.7 \pm 6.5$ \\
\hline FSH (mIU/mL) & $2-11$ & $14.6 \pm 5.5$ & $8.0 \pm 5.0$ \\
\hline $\mathrm{LH}(\mathrm{mIU} / \mathrm{mL})$ & $2-11$ & $11.9 \pm 8.7$ & $12.1 \pm 8.2$ \\
\hline Estradiol (pg/mL) & $11-110$ & $41.3 \pm 44.5$ & $68.5 \pm 66.5$ \\
\hline Progesterone (pg/mL) & $0-20$ & $3.9 \pm 8.2$ & $3.1 \pm 4.4$ \\
\hline Testosterone $(\mathrm{ng} / \mathrm{mL})^{*}$ & $0.2-0.6$ & $0.2 \pm 0.2$ & $0.5 \pm 1.2$ \\
\hline Valproate serum level (ng/mL) & $50-100$ & $50.6 \pm 14.8$ & $57.4 \pm 17.4$ \\
\hline
\end{tabular}

${ }^{*} \mathrm{p}<0.05$. ALP: alkaline phosphatase, DEXA: dual-energy X-ray absorptiometry, FSH: follicle-stimulation hormone, LH: luteinizing hormone

including BMI, group (subjects with either abnormal vs. normal DEXA scans), phosphorus, and osteocalcin, had an effect on the femur and lumbar BMD values ( $p>0.05)$.

\section{DISCUSSION}

These results suggest that long-term treatment with valproate combined with a low-dose atypical antipsychotic may adversely affect BMD and bone metabolism in premenopausal women with bipolar disorder. Decreased BMD was more prominent in the proximal femur than in the lumbar spine, and the biochemical markers that reflect bone metabolism may be related to osteoporosis or osteopenia.

The most common form of bone loss is postmenopausal osteoporosis related to estrogen deficiency. A main cause of secondary osteoporosis is disease-induced or drug-induced bone loss. ${ }^{5}$ Studies associated with BMD in psychiatry have focused mainly on schizophrenic patients. In women suffering from schizophrenia, the associated antipsychotic-induced hyperprolactinemia, hypogonadism, and resulting estrogen deficiency may reduce bone density. ${ }^{15}$ All conventional and atypical antipsychotics, which are potent $\mathrm{D}_{2}$ inhibitors and cause prolactin elevation, have been associated with decreased BMD. ${ }^{6,16}$ However, there is some controversy as to whether the medication or the disease itself causes the hyperprolactinemia and decreased BMD. ${ }^{3,5,6,17,18}$ In depression, elevation in cortisol levels contributes to low BMD through decreased bone turnover, and low estrogen level is associated with decreased BMD. ${ }^{4,5}$ However, few studies have examined the relationship between bipolar disorder or medication for this disease and
BMD. In one study, $60 \%$ of chronic psychiatric inpatients who were maintained on long-term valproate treatment had osteoporosis or osteopenia, although the diagnoses of these patients were not mentioned. ${ }^{19}$ In our results, almost half of the subjects showed decreased BMD with long-term valproate treatment combined with a low-dose atypical antipsychotic in premenopausal women with bipolar disorder. Previous studies have suggested that a reduction in BMD in epileptic patients using enzyme-inducing anticonvulsants was caused by an increase in vitamin D catabolism. ${ }^{9,10,20,21}$ Valproate may also reduce $\mathrm{BMD}$ by increasing vitamin $\mathrm{D}$ catabolism or affecting bone mineralization. ${ }^{19,22}$ However, valproate is not a hepatic enzyme inducer and therefore the decrease in bone density is expected to occur via another mechanism., ${ }^{8,22,23}$ Valproate may reduce BMD by inducing reversible Fanconi syndrome, causing renal tubular dysfunction resulting in excess calcium loss. ${ }^{9,22}$ Lack of correlation between vitamin D levels and BMD in our study may support a non-vitamin D-mediated mechanism of action for valproate on the skeleton as has been previously suggested. ${ }^{21,22}$ In addition to valproate, our subjects also took antipsychotics that can cause hyperprolactinemia, which may further increase their risk for low BMD. However, the level of prolactin was within the normal range $(18.57 \pm 10.68 \mathrm{ng} / \mathrm{mL})$ in all subjects, ${ }^{18}$ and comparison between the subjects with abnormal and normal DEXA scans did not show significant differences. Therefore, hyperprolactinemia cannot be a sufficient explanation for decreased BMD.

In our subjects, BMD was lower in the proximal femur than that in the lumbar spine. Valproate treatments in women with epilepsy have reduced BMD at the femoral neck, but not at the 
lumbar spine. ${ }^{20}$ Moreover, trabecular bone rather than cortical BMD may be more affected in patients who are taking valproate for bipolar disorder. This would be similar to the results demonstrating lower density of the trabecular bone but not cortical bone in patients with depressive disorder. ${ }^{4}$

Comparison between the two groups by BMD scores showed no significant difference in any clinical parameter. Subjects with abnormal DEXA scans showed a relatively longer duration of medication, however the correlation was not significant. Our subjects took their medications for over 2 years. Previous studies have suggested an association between the duration of valproate medication and osteoporosis. ${ }^{8,20,22,23}$ Therefore, a more comprehensive study will be needed to address this conflicting observation.

For all subjects, the mean for all biochemical makers were within normal ranges. In comparison, subjects with abnormal DEXA scans showed a significant decrease in phosphorous levels and tended to have a decrease in osteocalcin levels. Osteocalcin, which is produced by osteoblasts, is a marker protein of bone matrix synthesis and also plays a key role in the regulation of bone resorption. Oner et al..$^{23}$ found high normal osteocalcin levels in patients receiving valproate monotherapy in epilepsy patients. Our results suggest that phosphorus and osteocalcin may be related with bone loss in female bipolar patients treated with valproate and atypical antipsychotics. Interestingly, only testosterone showed a significantly lower level in subjects with abnormal DEXA scans relative to normal subjects. Androgens, which are similar to estrogen, have also been shown to indirectly influence bone metabolism by several potentially interrelated mechanisms. Due to its effect on osteoblastic activity, low levels of testosterone are correlated with osteoporosis in schizophrenic patients. ${ }^{24}$ In addition, Halbreich et al. ${ }^{3}$ found a correlation between BMD and testosterone in medicated men with psychiatric illnesses. Therefore, testosterone may play a role in low bone density.

The limitations of our report include its cross-sectional design rather than the preferred prospective, double blind study. The relatively small number of subjects is also a drawback. In addition, those patients taking valproate also were taking atypical antipsychotics that could increase serum prolactin, which may further increase their risk for low BMD. Studying BMD in patients with psychiatric illness may be much more difficult than studies in other populations (e.g. epilepsy) because mood stabilizers are frequently combined with antipsychotics in patients with bipolar disorder in clinical practice. We were unable to adjust for other potential confounders including race, although our study excluded as many confounding variables as possible.

In conclusion, this is the first report describing decreased $\mathrm{BMD}$ and changed biochemical markers of bone turnover in premenopausal bipolar patients on maintenance treatment with valproate and a low-dose atypical antipsychotic. Longterm maintenance treatment with valproate combined with a low-dose atypical antipsychotic may adversely affect BMD in premenopausal women with bipolar disorder. All bone metabolism biochemical markers were within normal ranges; as such we could not find an association between low BMD and biochemical makers of bone metabolism. Additional prospective studies with a larger population are warranted to better understand the exact mechanism of low BMD and biochemical changes in bipolar patients treated with valproate and atypical antipsychotics.

\section{REFERENCES}

1. Furlan PM, Ten Have T, Cary M, Zemel B, Wehrli F, Katz IR, et al. The role of stress-induced cortisol in the relationship between depression and decreased bone mineral density. Biol Psychiatry 2005;57:911-917.

2. Gold DT, Solimeo S. Osteoporosis and depression: a historical perspective. Curr Osteoporos Rep 2006;4:134-139.

3. Halbreich U, Rojansky N, Palter S, Hreshchyshyn M, Kreeger J, Bakhai $\mathrm{Y}$, et al. Decreased bone mineral density in medicated psychiatric patients. Psychosom Med 1995;57:485-491.

4. Michelson D, Stratakis C, Hill L, Reynolds J, Galliven E, Chrousos G, et al. Bone mineral density in women with depression. N Engl J Med 1996;335:1176-1181.

5. Misra M, Papakostas GI, Klibanski A. Effects of psychiatric disorders and psychotropic medications on prolactin and bone metabolism. J Clin Psychiatry 2004;65:1607-1618.

6. Meaney AM, O'Keane V. Bone mineral density changes over a year in young females with schizophrenia: relationship to medication and endocrine variables. Schizophr Res 2007;93:136-143.

7. Soares CN, Taylor V. Effects and management of the menopausal transition in women with depression and bipolar disorder. J Clin Psychiatry 2007;68(Suppl 9):16-21.

8. Sato Y, Kondo I, Ishida S, Motooka H, Takayama K, Tomita Y, et al. Decreased bone mass and increased bone turnover with valproate therapy in adults with epilepsy. Neurology 2001;57:445-449.

9. Sheth RD, Wesolowski CA, Jacob JC, Penney S, Hobbs GR, Riggs JE, et al. Effect of carbamazepine and valproate on bone mineral density. J Pediatr 1995; 127:256-262.

10. Stephen LJ, McLellan AR, Harrison JH, Shapiro D, Dominiczak MH, Sills GJ, et al. Bone density and antiepileptic drugs: a case-controlled study. Seizure 1999;8:339-342.

11. Triantafyllou N, Lambrinoudaki I, Armeni E, Evangelopoulos EM, Boufidou F, Antoniou A, et al. Effect of long-term valproate monotherapy on bone mineral density in adults with epilepsy. J Neurol Sci 2010;290:131-134.

12. WHO Scientific Group on the Prevention and Management of Osteoporosis. Prevention and Management of Osteoporosis: Report of a WHO Scientific Group (WHO Technical Report Series; 921). Geneva, Switzerland: World Health Organization; 2000.

13. Koh KC, Sohn HK, Kim YP, Ham KS, Chun BO, Lee JM. The study on bone mineral density use for DEXA in korean adults women. Korea J Sports Sci 2000;9:817-830.

14. Park SO, Lee IJ, Shin GS. The relationship of age, body mass index, and individual habit to bone mineral density in adults. J Korean Soc Radiol Sci 2008;31:367-377.

15. Klibanski A, Neer RM, Beitins IZ, Ridgway EC, Zervas NT, McArthur JW. Decreased bone density in hyperprolactinemic women. N Engl J Med 1980;303:1511-1514. 
16. O'Keane V, Meaney AM. Antipsychotic drugs: a new risk factor for osteoporosis in young women with schizophrenia? J Clin Psychopharmacol 2005;25:26-31.

17. Abraham G, Friedman RH, Verghese C. Osteoporosis demonstrated by dual energy $\mathrm{x}$-ray absorptiometry in chronic schizophrenic patients. Biol Psychiatry 1996;40:430-431.

18. Canuso CM, Goldstein JM, Wojcik J, Dawson R, Brandman D, Klibanski A, et al. Antipsychotic medication, prolactin elevation, and ovarian function in women with schizophrenia and schizoaffective disorder. Psychiatry Res 2002;111:11-20.

19. Waheed A, Kettl PA. Low bone density with the use of valproate. Gen Hosp Psychiatry 2005;27:376-378.

20. Farhat G, Yamout B, Mikati MA, Demirjian S, Sawaya R, El-Hajj Fuleihan G. Effect of antiepileptic drugs on bone density in ambulatory pa- tients. Neurology 2002;58:1348-1353.

21. Kim SH, Lee JW, Choi KG, Chung HW, Lee HW. A 6-month longitudinal study of bone mineral density with antiepileptic drug monotherapy. Epilepsy Behav 2007;10:291-295.

22. Boluk A, Guzelipek M, Savli H, Temel I, Ozisik HI, Kaygusuz A. The effect of valproate on bone mineral density in adult epileptic patients. Pharmacol Res 2004;50:93-97.

23. Oner N, Kaya M, Karasalihoglu S, Karaca H, Celtik C, Tutunculer F. Bone mineral metabolism changes in epileptic children receiving valproic acid. J Paediatr Child Health 2004;40:470-473.

24. Bergemann N, Parzer P, Mundt C, Auler B. High bone turnover but normal bone mineral density in women suffering from schizophrenia. Psychol Med 2008;38:1195-1201. 